\title{
Solution of a Linear Pursuit-Evasion Differential Game with Closed and Convex Terminal Set
}

\author{
Gafurjan I. Ibragimov ${ }^{1}$, Marzieh Khakestari ${ }^{2} \&$ Atamurat Sh. Kuchkarov ${ }^{2,3}$ \\ ${ }^{1}$ INSPEM and Department of Mathematics, Faculty of Science, \\ Universiti Putra Malaysia, Serdang, Malaysia \\ ${ }^{2}$ Institute for Mathematical Research, Universiti Putra Malaysia, Serdang, Malaysia \\ ${ }^{3}$ Institute of Mathematics and Information Technology of Academy of Sciences of \\ Uzbekistan, 100125, Durman yuli, 29, Tashkent, Uzbekistan \\ Email: marzieh@inspem.upm.edu.my
}

\begin{abstract}
A linear two-person zero-sum pursuit-evasion differential game is considered. Control functions of players are subject to integral constraints. Terminal set is a closed convex subset of $\square^{n}$. The Pursuer tries to bring the state of the system to the terminal set and the Evader prevents bringing of the state to the terminal set where control resource of the Pursuer is greater than that of Evader. We obtain a formula for the optimal pursuit time and construct optimal strategies of the players in explicit form.
\end{abstract}

Keywords: control; pursuit-evasion game; optimal pursuit time; strategy; terminal set.

\section{Introduction}

We study a linear pursuit-evasion differential game. Such games are investigated by many researchers [1-14]. Below we review some of them.

Pshenichnii and Onopchuk [1] considered a linear differential game with integral constraints on controls of the players:

$$
\dot{z}=A z+b u-c v,
$$

where $A$ is a constant $n \times n$ matrix, $b$ and $c$ are $n$ vectors, $u$ and $v$ are scalar control parameters of the Pursuer and Evader, respectively. The terminal set $M$ is a closed convex manifold. Some conditions to complete the game for optimal pursuit time were obtained.

Mezentsev [2] studied a pursuit-evasion differential game described by the equation

$$
\dot{z}=A(t) z-B(t) u+C(t) v,
$$

Received January $10^{\text {th }}, 2011$, Revised April $19^{\text {th }}, 2011$, Accepted for publication July $25^{\text {th }}, 2011$.

Copyright $@ 2012$ Published by LPPM ITB, ISSN: 1978-3043, DOI: 10.5614/itbj.sci.2012.44.1.1 
where $A(t)$ is $n \times n$ matrix, $u \in \square^{p}$ and $v \in \square^{q}$ are control parameters of the Pursuer and Evader, $B(t)$ and $C(t)$ are $n \times p$ and $n \times q$ matrices, and $A(t), B(t)$ and $C(t), \mathrm{t} \geq 0$ are continuous. The terminal set $M$ is a closed convex subspace of $\square^{n}$. It was obtained sufficient conditions for terminating of pursuit from the given initial point. Moreover, the guaranteed time for termination of pursuit was studied.

Nikolskii [3] investigated linear pursuit-evasion differential games under geometric constraints. A method for solving the pursuit-evasion game was presented where the terminal set is closed and convex.

Note that Ibragimov [4] studied an optimal pursuit game with one Pursuer and one Evader in a Hilbert space. Also Ibragimov [5] constructed optimal strategies for players and found a formula for the optimal pursuit time.

Azimov [6] investigated a linear pursuit differential game under integral constraints and obtained sufficient conditions for evasion from any point of the phase space. Azimov [7] devoted his work to the linear evasion differential games with integral constraints and acquired sufficient conditions for escape from any given point of the phase space.

Azamov [8] examined in depth some simple motions differential games.

In this study, we solve a linear pursuit-evasion differential game problem with integral constraints on control functions. Assume that the control resource of the Pursuer is greater than that of the Evader. The differential game is governed by the equation

$$
\dot{z}(t)=A(t) z+B(t)(v-u), \quad z(0)=z_{0},
$$

where $z \in \square^{n}, z_{0} \in \square^{n} \backslash M$ and $u, v \in \square^{n}$ are control parameters of the Pursuer and Evader, respectively, $A(t)$ and $B(t)$ are continues $n \times n$ matrices. The game is ended when the trajectory $z$ reaches given terminal set $M$. We suppose that $M$ is a given closed convex subset of the state space $\square^{n}$. The minimizing player, the Pursuer, tries to realize the event $z(t) \in M$ as earlier as possible, while the maximizing player, the Evader, pursues the opposite aim.

\section{Definition 1}

A measurable function $u:[0, \infty) \rightarrow \square^{n}\left(v:[0, \infty) \rightarrow \square^{n}\right)$ subject to 


$$
\int_{0}^{\infty}|u(t)|^{2} d t \leq \rho^{2} \quad\left(\int_{0}^{\infty}|v(t)|^{2} d t \leq \sigma^{2}\right)
$$

is called a control of the Pursuer $P$ (the Evader $E$ ), where $\rho$ and $\sigma$ are given positive numbers $(\rho>\sigma)$. We denote by $U_{\rho}$ (respectively $V_{\sigma}$ the set of all controls of the Pursuer $P$ (Evader $E$ ).

\section{Definition 2}

A function $U(t, v), U:[0, \infty) \times \square^{n} \rightarrow \square^{n}$, is called a strategy of the Pursuer if for any admissible control $v(\cdot) \in V_{\sigma}$ of the Evader, the system

$$
\dot{z}(t)=A(t) z+B(t)\left(v(t)-U(t, v(t)), \quad z(0)=z_{0},\right.
$$

has a unique solution and

$$
\int_{0}^{\infty}|U(v(t))|^{2} d t \leq \rho^{2}
$$

\section{Definition 3}

A function $V(t, u, \varepsilon), \quad V:[0, \infty) \times \square^{n} \times \square^{+} \rightarrow \square^{n}$, of the form

$$
\begin{aligned}
& V(t, u, \varepsilon)=0, \quad 0 \leq t \leq \varepsilon, \\
& V(t, u, \varepsilon)=F(t, \varepsilon) u(t-\varepsilon), \quad t>\varepsilon,
\end{aligned}
$$

where $u(\cdot) \in U_{\rho}, \quad F(t, \varepsilon)$ is a matrix, $\varepsilon$ is a positive number, and $\int_{0}^{\infty}|V(t, u(t), \varepsilon)|^{2} d t \leq \sigma^{2}$, is called a strategy of the Evader.

\section{Definition 4}

A finite number $T^{*}$ is called optimal pursuit time if the following two conditions hold:

1. there is a strategy $U_{0}$ of the Pursuer such that for any $v(\cdot) \in V_{\sigma}$ the inclusion $z\left(t, z_{0}, U_{0}, v(\cdot)\right) \in M$ holds at some $t \in\left[0, T^{*}\right]$. In this case, we say that pursuit can be completed for the time $T^{*}$.

2. for any $h_{0}, 0<h_{0}<T^{*}$, there is a strategy $V_{0}$ of the Evader such that $z\left(t, z_{0}, u(\cdot), V_{0}\right) \notin M$ for any control $u(\cdot) \in U_{\rho}$ and $t \in\left[0, T^{*}-h_{0}\right]$. In this case we say that the evasion is possible on $\left[0, T^{*}\right)$. If for any $T>0$ evasion is possible on $[0, T)$, then we say that evasion is possible on $[0, \infty)$. 
The strategies $U_{0}$ and $V_{0}$ are called optimal strategies of the Pursuer and Evader, respectively.

The problem is to construct optimal strategies of the players and find optimal pursuit time in the game (1), (2).

\section{Auxiliary Results}

The equation (1) has the solution

$$
z(t)=\Psi(t) y(t), \quad t \geq 0,
$$

where $\Psi(t)$ is the fundamental matrix of the equation $\dot{z}(t)=A(t) z$

satisfying $\Psi(0)=E$,

$$
y(t)=z_{0}+\int_{0}^{t} \Psi^{-1}(s) B(s)(v(s)-u(s)) d s .
$$

We obtain

$$
\dot{y}=C(t)(v(t)-u(t)), y(0)=z_{0},\left(C(t)=\Psi^{-1}(t) B(t)\right) .
$$

For any $t \geq 0$, we suppose that $\operatorname{det} B(t) \neq 0$, and hence $\operatorname{det} C(t) \neq 0$. We define a nonempty set $M(t) \subset \square^{n}$ by the formula

$$
M(t)=\Psi^{-1}(t) M .
$$

Note that for the trajectory $z(t)$ of the system (1), $z(t) \in M$ at some $t \geq 0$ if and only if $y(t) \in M(t) . M$ is closed and convex set and so is $M(t)$.

Thus, we have obtained an equivalent differential game given by the equation (3) and with the terminal set $M(t)$.

To construct strategy of the Pursuer we need to define an attainability set for a controlled system. We consider the following system

$$
\dot{y}=C(t) w(t), y(0)=z_{0},
$$

which is obtained from (3) by substituting $u(t)-v(t)=w(t)$.

Let

$$
F(\theta)=\int_{0}^{\theta} C(s) C^{*}(s) d s
$$


It is clear that the matrix $F(\theta)$ is symmetric. Where $C^{*}$ is the transpose of the matrix $C$. It can be shown easily that the matrix $F(t), t>0$, is positive definite. Therefore, the inverse matrix $F^{-1}(t)$ exists.

We obtain from (4)

$$
y(\theta)=z_{0}+\int_{0}^{\theta} C(s) w(s) d s .
$$

The attainability set for the system (4) from the initial position $y(0)=z_{0}$ to the time $\theta$, is

$$
X(\theta)=\left\{y(\theta) \in \square^{n}: y(\theta)=z_{0}+\int_{0}^{\theta} C(s) w(s) d s\right\},
$$

where the measurable control function $w(t), 0 \leq t \leq \theta$, satisfies the inequality

$$
\left(\int_{0}^{\theta}|w(s)|^{2}\right)^{1 / 2} \leq \rho-\sigma
$$

It is known (Krasovskii [9]) that $X(\theta)$ is an ellipsoid.

\subsection{Construction of Attainability Set}

We use the following (see Lee and Markus [10])

Assertion 1 Let $C(t), 0 \leq \mathrm{t} \leq \vartheta$, be a continuous $n \times n$ matrix, and its determinant be not identically 0 on $[0, \vartheta]$. Then among the measurable functions $w(\cdot), w:[0, \vartheta] \rightarrow \square^{n}$, satisfying the condition

$$
\int_{0}^{\vartheta} C(s) w(s) d s=\xi
$$

the control $w(\cdot)$ defined almost everywhere on $[0, \vartheta]$ by the formula

$$
w(s)=C^{*}(s) F^{-1}(\vartheta) \xi, F(\vartheta)=\int_{0}^{\vartheta} C(s) C^{*}(s) d s,
$$

gives the minimum to the functional

$$
\int_{0}^{\vartheta}|w(s)|^{2} d s
$$


According to the Assertion if we let $\xi=y(\theta)-z_{0} \quad$ (see (5)) and $\vartheta=\theta$, then among the controls satisfying (5) the control

$$
w(s)=C^{*}(s) F^{-1}(\theta)\left(y(\theta)-z_{0}\right)
$$

gives the minimum to the functional

$$
\int_{0}^{\theta}|w(s)|^{2} d s
$$

Hence, according to (5) and (7) we have

$$
\begin{aligned}
\int_{0}^{\theta}|w(s)|^{2} d s & =\int_{0}^{\theta}\langle w(s), w(s)\rangle d s \\
& =\int_{0}^{\theta}\left\langle C^{*}(s) F^{-1}(\theta)\left(y(\theta)-z_{0}\right), C^{*}(s) F^{-1}(\theta)\left(y(\theta)-z_{0}\right)\right\rangle d s \\
& =\int_{0}^{\theta}\left\langle C(s) C^{*}(s) F^{-1}(\theta)\left(y(\theta)-z_{0}\right), F^{-1}(\theta)\left(y(\theta)-z_{0}\right)\right\rangle d s \\
& =\left(y(\theta)-z_{0}\right) F^{-1}(\theta)\left(y(\theta)-z_{0}\right) \leq(\rho-\sigma)^{2},
\end{aligned}
$$

where $\langle x, y\rangle$ is the inner product of vectors $x$ and $y$. Note that the set (6) can be defined as follows

$$
X(\theta)=\left\{y(\theta) \in \square^{n}:\left(y(\theta)-z_{0}\right) F^{-1}(\theta)\left(y(\theta)-z_{0}\right) \leq(\rho-\sigma)^{2}\right\} .
$$

It should be noted that $X(\theta)$ is a closed ellipsoid with the center $z_{0}$.

\subsection{Construction of a control}

We consider the controlled system (4) and show that any point $\bar{y}$ of $X(\theta)$ is reachable. Indeed, let $\bar{y} \in X(\theta)$ and

$$
w_{0}(t)= \begin{cases}C^{*}(t) F^{-1}(\theta)\left(\bar{y}-z_{0}\right), & 0 \leq t \leq \theta, \\ 0 & t>\theta .\end{cases}
$$

We show that $y(\theta)=\bar{y}$. Indeed,

$$
\begin{aligned}
& y(\theta)=z_{0}+\int_{0}^{\theta} C(s) w_{0}(s) d s \\
& =z_{0}+\int_{0}^{\theta} C(s) C^{*}(s) F^{-1}(\theta)\left(\bar{y}-z_{0}\right) d s \\
& =z_{0}+\bar{y}-z_{0}=\bar{y}
\end{aligned}
$$


We now show admissibility of (9). We obtain

$$
\begin{aligned}
& \int_{0}^{\infty}\left|w_{0}(t)\right|^{2} d t=\int_{0}^{\theta}\left|C^{*}(t) F^{-1}(\theta)\left(\bar{y}-z_{0}\right)\right|^{2} d t \\
& =\int_{0}^{\theta}\left\langle C^{*}(t) F^{-1}(\theta)\left(\bar{y}-z_{0}\right), C^{*}(t) F^{-1}(\theta)\left(\bar{y}-z_{0}\right)\right\rangle d t \\
& =\int_{0}^{\theta}\left\langle C(t) C^{*}(t) F^{-1}(\theta)\left(\bar{y}-z_{0}\right), F^{-1}(\theta)\left(\bar{y}-z_{0}\right)\right\rangle d t \\
& =\left(\bar{y}-z_{0}\right) F^{-1}(\theta)\left(\bar{y}-z_{0}\right) \leq(\rho-\sigma)^{2}
\end{aligned}
$$

\section{Main Results}

We shall use the following Lemma.

Lemma 1 If $t_{1}<t_{2}$, then $X\left(t_{1}\right) \subset X\left(t_{2}\right)$.

Proof Let $y \in X\left(t_{1}\right)$, then by the definition of (8), we have

$$
\left(y-z_{0}\right) F^{-1}\left(t_{1}\right)\left(y-z_{0}\right) \leq(\rho-\sigma)^{2} .
$$

It is not difficult to verify that the function $f(t)=\left(y-z_{0}\right) F^{-1}(t)\left(y-z_{0}\right)$ is decreasing. Therefore, for any $t_{1}<t_{2}$, we have $f\left(t_{2}\right)<f\left(t_{1}\right)$, hence

$$
\left(y-z_{0}\right) F^{-1}\left(t_{2}\right)\left(y-z_{0}\right) \leq(\rho-\sigma)^{2} .
$$

It follows that $y \in X\left(t_{2}\right)$. This completes the proof of Lemma 1.

Theorem 1 Let $\rho>\sigma$, and there exists a number $\theta$, such that

$$
\theta=\min \{t \mid X(t) \cap M(t) \neq \varnothing\} .
$$

Then $\theta$ is the optimal pursuit time in the game (3).

Proof $1^{0}$. Proof that pursuit can be completed for the time $\theta$. As $X(t)$ and $M(t)$ are closed and so is their intersection. Hence, $\theta$ exists. According to hypothesis of the theorem $X(\theta) \cap M(\theta) \neq \varnothing$. Then there exists $\bar{y} \in X(\theta) \cap M(\theta)$. Hence, $\bar{y} \in X(\theta)$ and $\bar{y} \in M(\theta)$. We define the strategy of the Pursuer as follows 


$$
U(t, v(t))=\left\{\begin{array}{lc}
v(t)-C^{*}(t) F^{-1}(\theta)\left(\bar{y}-z_{0}\right), & 0 \leq t \leq \theta, \\
0 & t>\theta .
\end{array}\right.
$$

We now show admissibility of the strategy. Indeed, by using the Minkowski inequality, and the fact that $\bar{y} \in X(\theta)$, we get

$$
\begin{aligned}
\left(\int_{0}^{\infty}|U(t, v(t))|^{2} d t\right)^{1 / 2} & =\left(\int_{0}^{\theta}\left|v(t)-C^{*}(t) F^{-1}(\theta)\left(\bar{y}-z_{0}\right)\right|^{2} d t\right)^{1 / 2} \\
& \leq\left(\int_{0}^{\theta}|v(t)|^{2} d t\right)^{1 / 2}+\left(\int_{0}^{\theta}\left|C^{*}(t) F^{-1}(\theta)\left(\bar{y}-z_{0}\right)\right|^{2} d t\right)^{1 / 2} \\
& \leq \sigma+\rho-\sigma=\rho .
\end{aligned}
$$

Therefore, $U(\cdot, v(\cdot)) \in U_{\rho}$.

We now show that pursuit can be completed at the time $\theta$. By (11)

$$
\dot{y}=C(t)(v(t)-U(t, v(t)))=C(t) C^{*}(t) F^{-1}(\theta)\left(\bar{y}-z_{0}\right) .
$$

Hence,

$$
y(\theta)=z_{0}+\int_{0}^{\theta} C(t) C^{*}(t) F^{-1}(\theta)\left(\bar{y}-z_{0}\right) d t=z_{0}+\left(\bar{y}-z_{0}\right)=\bar{y} \in M(\theta) .
$$

So pursuit is completed at $\theta$.

$2^{0}$. At this point we have to show that for any fixed $h_{0} \in(0, \theta)$ there exists a strategy of the Evader such that evasion is possible on $\left[0, \theta-h_{0}\right)$. Let

$$
2 \varepsilon=\min _{0<t \leq \theta-h_{0}} d(X(t), M(t)) .
$$

By definition of $\theta, X(t) \cap M(t)=\varnothing, 0 \leq t \leq \theta-h_{0}$, and the sets $X(t)$ and $M(t)$ are closed convex therefore $\varepsilon>0$.

Let

$$
S_{\varepsilon}=\left\{x\left|x \in \square^{n},\right| x \mid<\varepsilon\right\},
$$

i.e. $S_{\varepsilon}$ is an open ball with the center at the origin and radius $\varepsilon$. We choose a number $\varepsilon_{1}$ such that

$$
\left(\int_{0}^{h}\|C(s)\|^{2} d s\right)^{1 / 2} \frac{\sigma \varepsilon_{1}}{\left(1+\varepsilon_{1}\right)}<\frac{\varepsilon}{2}
$$


We now choose $h_{1}, 0<h_{1}<h_{0}$, such that

$$
\left\|C^{-1}(t) C(t-h)\right\| \leq 1+\varepsilon_{1}, \quad h \leq t \leq \theta, \quad 0<h<h_{1} .
$$

We construct the strategy $V$ for the Evader as follows

$$
V(t)= \begin{cases}0, & 0 \leq t<h \\ \alpha C^{-1}(t) C(t-h) u(t-h), & h \leq t \leq \theta, \\ 0, & t>\theta .\end{cases}
$$

where $0<h<h_{1}$

$$
\alpha=\frac{\sigma}{\left(1+\varepsilon_{1}\right) \rho} .
$$

This strategy is admissible, since by (12)

$$
\begin{aligned}
\int_{0}^{\theta}|v(t)|^{2} d t & =\int_{0}^{h}|v(t)|^{2} d t+\int_{h}^{\theta}|v(t)|^{2} d t \\
& \leq \frac{\sigma^{2}}{\left(1+\varepsilon_{1}\right)^{2} \rho^{2}} \int_{h}^{\theta}|| C^{-1}(t) C(t-h) \|\left.\right|^{2}|u(t-h)|^{2} d t \\
& \leq \frac{\sigma^{2}}{\left(1+\varepsilon_{1}\right)^{2} \rho^{2}}\left(1+\varepsilon_{1}\right)^{2} \int_{h}^{\theta}|u(t-h)|^{2} d t \\
& \leq \frac{\sigma^{2}}{\rho^{2}} \int_{0}^{\theta-h}|u(t)|^{2} d t \leq \frac{\sigma^{2}}{\rho^{2}} \rho^{2}=\sigma^{2} .
\end{aligned}
$$

Hence, strategy (13) is admissible.

We have

$$
\begin{aligned}
y(t)=z_{0} & +\int_{0}^{t} C(s)(u(s)-V(s)) d s=z_{0}+\int_{0}^{t} C(s) u(s) d s-\alpha \int_{h}^{t} C(s-h) u(s-h) d s \\
& =z_{0}+\int_{0}^{t} C(s) u(s) d s-\alpha \int_{0}^{t-h} C(s) u(s) d s \\
& =z_{0}+(1-\alpha) \int_{0}^{t-h} C(s) u(s) d s+\int_{t-h}^{t} C(s) u(s) d s .
\end{aligned}
$$

For a set $A$ we denote $A_{\varepsilon}=A+S_{\varepsilon}$. If we show the inclusion

$$
y(t) \in X_{2 \varepsilon}(t), \quad 0 \leq t \leq \theta-h_{0},
$$


10

Gafurjan I. Ibragimov, et al.

then we obtain $y(t) \notin M(t), 0 \leq t \leq \theta-h_{0}$, and the proof of the Theorem follows. We now show (16). Put

$$
w(s)=\frac{\rho-\sigma}{\rho} u(s), \quad 0 \leq t \leq \theta .
$$

Then for $t \in\left[0, \theta-h_{0}\right]$, we get from (15)

$$
\begin{aligned}
y(t) & =z_{0}+\frac{(1-\alpha) \rho}{\rho-\sigma} \int_{0}^{t-h} C(s) w(s) d s+\int_{t-h}^{t} C(s) u(s) d s \\
& =\left(\frac{(1-\alpha) \rho}{\rho-\sigma}-1\right) \int_{0}^{t-h} C(s) w(s) d s+\left(z_{0}+\int_{0}^{t-h} C(s) w(s) d s\right)+\int_{t-h}^{t} C(s) u(s) d s \\
& =I_{1}+I_{2}+I_{3},
\end{aligned}
$$

where

$$
\begin{aligned}
& I_{1}=\left(\frac{(1-\alpha) \rho}{\rho-\sigma}-1\right) \int_{0}^{t-h} C(s) w(s) d s, \\
& I_{2}=z_{0}+\int_{0}^{t-h} C(s) w(s) d s, \quad I_{3}=\int_{t-h}^{t} C(s) u(s) d s .
\end{aligned}
$$

As (see (14))

$$
\frac{(1-\alpha) \rho}{\rho-\sigma}-1=\frac{\sigma \varepsilon_{1}}{\left(1+\varepsilon_{1}\right)(\rho-\sigma)}
$$

and in the set of functions $w(\cdot)$ satisfying

$$
\int_{0}^{\theta}|w(s)|^{2} d s \leq(\rho-\sigma)^{2},
$$

we have

$$
\begin{aligned}
\left|\int_{0}^{t-h} C(s) w(s) d s\right| & \leq \int_{0}^{t-h}\|C(s)\| w(s) \mid d s \\
& \leq\left(\int_{0}^{t-h}\|C(s)\|^{2} d s\right)^{1 / 2}\left(\int_{0}^{t-h}|w(s)|^{2} d s\right)^{1 / 2} \\
& \leq\left(\int_{0}^{\theta}\|C(s)\|^{2} d s\right)^{1 / 2} \cdot(\rho-\sigma),
\end{aligned}
$$

then by the choice of $\varepsilon_{1}$ we have 


$$
I_{1} \in \frac{1}{2} S_{\varepsilon}
$$

Next, by definition of $X(t)$ and Lemma 1

$$
I_{2} \in X(t-h) \subset X(t) \subset X_{\varepsilon}(t) .
$$

As to $I_{3}$, we have

$$
\left|I_{3}\right| \leq\left(\int_{0}^{h}\|C(s)\|^{2} d s\right)^{1 / 2} \rho
$$

And hence $\left|I_{3}\right| \rightarrow 0$ as $h \rightarrow 0$. Therefore we can choose a number $h_{2}, 0<h_{2}<h_{1}$, such that $\left|I_{3}\right|<\frac{\varepsilon}{2}, 0<h<h_{2}$, hence,

$$
I_{3} \in \frac{1}{2} S_{\varepsilon}, \quad 0<h<h_{2} .
$$

From (17), (19), (20) and (21) we obtain

$$
y(t) \in X_{\varepsilon}(t)+S_{\varepsilon} \subset X_{2 \varepsilon}(t), \quad t \in\left[0, \theta-h_{0}\right], \quad 0<h<h_{2},
$$

for all $w(\cdot)$ satisfying (18). Then by definition of $\varepsilon$

$$
y(t) \notin M(t), \quad 0 \leq t \leq \theta-h_{0} .
$$

This completes the proof of the Theorem 1 .

Theorem 2 If $\rho>\sigma$ and $X(t) \cap M(t)=\varnothing, t \geq 0$, then evasion is possible on $[0, \infty)$.

Proof Let $T>0$ be any fixed number. According to hypothesis of the theorem, particularly, $X(t) \cap M(t)=\varnothing$ on $0 \leq t \leq T$. Further proof of the theorem is similar to the proof of the part $2^{0}$ of Theorem 1 with $\theta-h_{0}$ replaced by $T$.

\section{References}

[1] Pshenichnii, B.N. \& Onopchuk, Yu., N., Linear Differential Games with Integral Constraints, Izvestige Akademii Nauk SSSR, Tekhnicheskaya Kibernetika, 1, pp. 13-22, 1968.

[2] Mezentsev A.V., A Direct Method in Linear Differential Games with Different Constraints, Zh. vyhisl. Mat. Mat. Fiz. 11(2), pp. 365-374, 1971. 
[3] Nikolskii M.S., A Direct Method In Linear Differential Pursuit-Evasion Games, V. A. Steklov Mathematics Institute, Academy of Sciences of the USSR, 33(2), pp. 885-891, 1983.

[4] Ibragimov, G.I., A Problem of Optimal Pursuit in Systems with Distributed Parameter, J. Appl. Math. Mech., 66(5), pp. 719-724, 2003.

[5] Ibragimov, G.I., A Game Problem on A Closed Convex Set, Siberian Advances in Mathematics, 12(3), pp. 16-31, 2002.

[6] Azimov, A.Ya., Linear Differential Pursuit Games with Integral Constraints on The Control, Differentsial'nye Uravneniya 11, 1975, English transl. in Differential Equations, 11, pp. 1723-1731, 1975.

[7] Azimov, A.Ya., A Linear Differential Evasion Game with Integral Constraints on The Controls, Zh. Vychisl. Mat. Mat. Fiz, 14(6), pp. 1427-1436, 1974.

[8] Azamov, A.A., Samatov, B., $\pi$-Strategy, An Elementary Introduction to the Theory of Differential Games, National University of Uzbekistan, Tashkent, Uzbekistan, p. 32, 2000.

[9] Krasovskii, N.N., Upravlenie Dinamicheskoi Sistemoi (Control of a dynamical system), Moscow, 1985.

[10] Lee, E.B. \& Markus, L., Foundations of Optimal Control Theory, John Wiley \& Sons, Inc., New York, 1967.

[11] Hajek, O., Pursuit Games, Academic Press, New York-San Francisco, 1975.

[12] Krasovskii, N.N., Upravlenie Dinamicheskoi Sistemoi (Control of a Dynamical System), Moscow, 1985.

[13] Petrosyan, L.A., Differential Games of Pursuit, World Scientific, Singapore, London, 1993.

[14] Pontryagin, L.S. \& Mishchenko, E.F., On Evasion Problem of One Controlled Object from Another, Dokl. AN. SSSR., 189(4), 721-723, 1969. 